
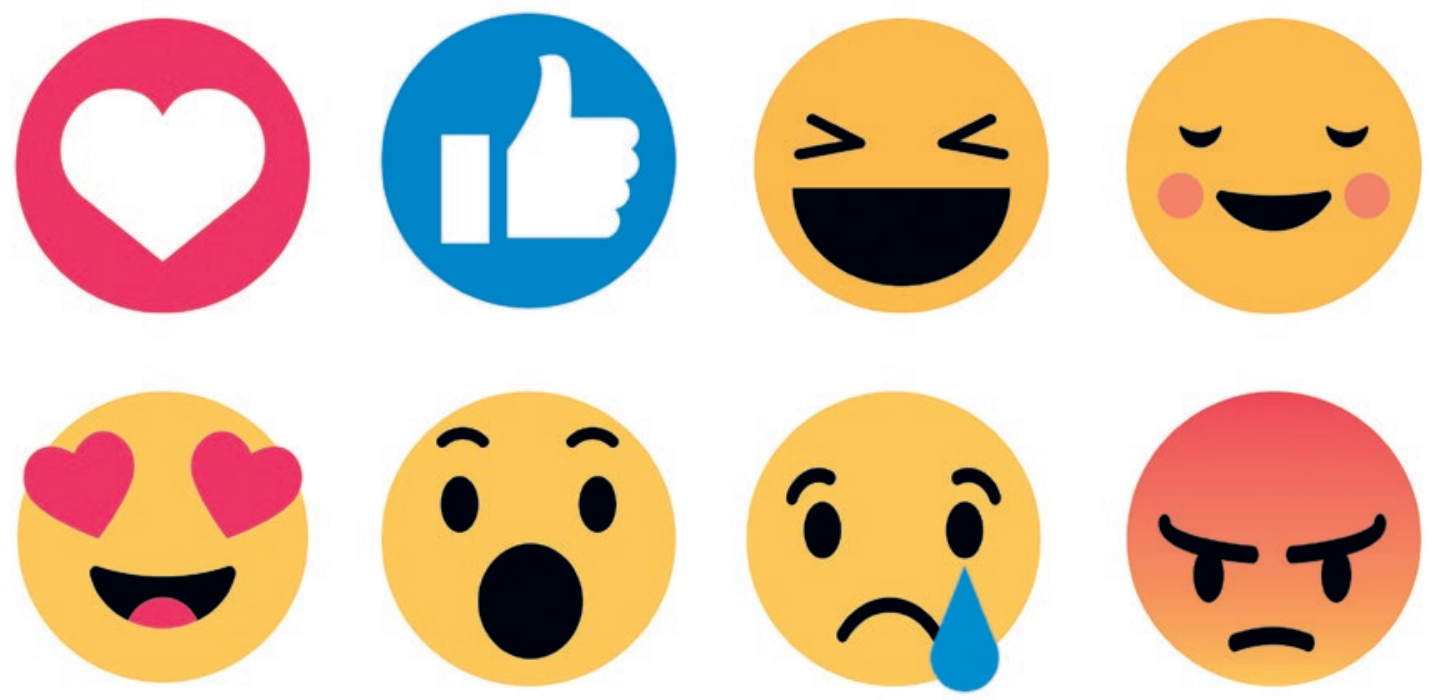

Symbole, die Befindlichkeiten ausdrücken und aus unserem Alltag beinahe nicht mehr wegzudenken sind: Emoticons.

\title{
Vom Symbol zur Diagnose
}

\section{Jann Schwarzenbach}

Dr. med., Facharzt für Allgemeinmedizin, Mitglied FMH

Wenn angehende Mediziner und Medizinerinnen beim Blick ins Mikroskop oder auf den Schirm des Ultraschallgeräts gar nichts sehen, so liegt das - glücklicherweise - keineswegs an einer pandemischen Retrobulbärneuritis, sondern an fehlenden wissensmässigen

Die Patienten werden damit gewissermassen zu Objektivierungen des berufswissenschaftlichen Denkens.

Orientierungspunkten. Erst durch unsere eigene geistige Leistung werden nämlich scheinbar zufällige Farbtupfen zum histologischen Präparat und ein chaotisches Geflimmer zum sonographischen Schnappschuss. Mit anderen Worten: Um dem ungeordneten Fluss sinnlicher Eindrücke Gestalt und Sinn abzugewinnen, müssen wir diesen erst einmal in unser eigenes Denken zwingen. Dazu benützen wir ein angelerntes Geflecht von Zeichen und Symbolen, die auf etwas Bestimmtes hindeuten beziehungsweise einen dahinterliegenden pathologischen Zusammenhang erschliessen. So steht dann eben eine unregelmässige Struktur für potenzielle Malignität, und ein Schallschatten wird zum Emblem des Gallensteinleidens. Aus einer ganzen
Fülle klinisch relevanter Wegmarken schaffen wir uns schlussendlich ein eigenes Bild der Menschen, die wir in der Sprechstunde vor uns haben. Die Letzteren werden damit gewissermassen zu Objektivierungen des berufswissenschaftlichen Denkens. Das mag zwar etwas einseitig und befremdlich erscheinen, macht aber durchaus Sinn: Es fördert nämlich den direkten Zugang zur schulmedizinischen Betreuung unserer Patienten.

\section{Emojis, Likes und Realität}

Nicht nur in der Heilkunde liesse sich über gedankliche Orientierungspunkte und Wegmarken spekulieren. Benötigen wir Menschen nicht ganz allgemein solche Hifsmittel, um uns im Dschungel der weltlichen Tatsachen und Geschehnisse erst einmal zurechtzufinden? Vielleicht haben wir ja gerade zu diesem Zweck den medizinischen Parametern nicht unähnlich - alle die verschiedenen Symbole wie Schriftzeichen, Zahlen, Laute, Emojis, Musiknoten, Likes und mythische Figuren erfunden. Diese könnten nämlich unentbehrlich sein, um unser Umfeld auf jeweils unterschiedliche, aber doch stets typisch menschliche Art zu erschlies- 
sen. Damit würde die krude Wirklichkeit, wie sie an sich ist, wohl aussen vor gelassen; dafür hätten wir uns aber eine eigene - durch unsere selbst in die Welt gebrachten Symbole bestimmte - Realität erschaffen.

\section{Laute, Emojis, Likes und mythische Figuren} könnten unentbehrlich sein, um unser Umfeld auf typisch menschliche Art zu erschliessen.

Wie eingangs am Beispiel unserer Patienten erläutert, könnte man diese Letztere als eine Art Objektivierung unserer geistigen Aktivität verstehen und ganz allgemein auch als Kultur bezeichnen. Das war jedenfalls die Ansicht von Ernst Cassirer (1874-1945), der überall auf der Welt symbolische Grundstrukturen postulierte: in der Wissenschaft, im Mythos, aber auch in der Sprache, in der Kunst und in der Religion. Im nächsten Abschnitt möchte ich nun versuchen, sein Denken ganz kurz zusammenzufassen.

\section{Vom «X» zu Kultur und Unkultur}

Als überzeugter Jünger seines geistigen Übervaters Immanuel Kant (1724-1804) verzichtet Cassirer auf jeden Anspruch, die Welt, so wie sie an sich sein mag, erkennen zu wollen. Sie bleibt für ihn das blosse "X», ein Chaos sinnlicher Wahrnehmungen, dem wir erst einmal eine gewisse Struktur geben müssen. Seiner Meinung nach tun wir dies mit Hilfe von selbsteingebrachten Zeichen und Symbolen. Dabei würden wir uns eine neue, private Aussenwelt erschaffen. Diese könnten wir dann auch - da sie ja eben eine Objektivierung der eigenen geistigen Tätigkeit sei - konkret erfahren, verstehen und beeinflussen. Eine solche hausgemachte Form von Realität sei nun keineswegs ein für alle Mal festgelegt, sondern sehe je nach Menschengruppe und geschichtlicher Epoche ganz anders aus. So sei denn im Laufe der Zeit eine Vielzahl menschengemachter Weltbilder entstanden, die Cassirer im weitesten Sinne eben auch als Kulturen bezeichnet. Er interessiert sich aber nun nicht primär dafür, wie diese Kulturen im Einzelnen aussehen mögen. Aufgabe der Philosophie sei es nämlich nicht, die Welt zu beschreiben, sondern die Bedingungen aufzusuchen, die ihr Erscheinen in einer verstehbaren Art möglich machen: die verschiedenartigen Zeichen und Symbole eben. Cassirers Ansicht nach sind diese nicht einfach arbiträr ausgewählte Phantasieprodukte. Sie stünden ganz allgemein für zeitlich-räumliche und qualitative
Einordnungen sowie für kausale Abhängigkeitsbestimmungen. Dies gelte beispielsweise ebenso für die Bildzeichen des Mythos wie auch für die Parameter der Naturwissenschaften. So bestehe, genau gesehen, zwischen den mythologischen und den zeitgenössischen Weltbildern kein prinzipieller, sondern nur ein gradueller Unterschied. Dieser Umstand berge - gerade in Zeiten des Umbruchs und einer allgemeinen Verunsicherung - ein stetes Risiko, in archaische Denkweisen zurückzufallen: in polarisierende Abgrenzungen und servierbereite Lösungen. Das klingt angesichts des zeitgenössischen digitalen Sumpfs schon fast prophetisch und wurde für Cassirer zur brutalen Realität. Als Angehöriger der jüdischen Glaubensgemeinschaft verlor er nämlich 1933 seine Rektoratsstelle in Hamburg und verliess kurz darauf das Land, das die humanistischen Symbole zunehmend in mythisch raunende und rassistisch schreiende Sinnbilder zurücktauschte, und mit dieser Unkultur seine grossen Denker - von Kant bis hin zu Nietzsche - verriet und missbrauchte.

\section{Der Arzt, das «Animal symbolicum»}

Die Art der Zeichen und Symbole, die wir produzieren und verwenden - für Cassirer ist der Mensch ein «Animal symbolicum» -, determinieren also in einem gewissen Sinne die Beschaffenheit der Welt, in der wir leben beziehungsweise leben wollen oder leben müssen. Das betrifft auch uns Ärzte und Ärztinnen. Nebst den eingangs beschriebenen, wissenschaftlich vorgegebe-

Symbole stehen für die Art von Medizin, die wir schlussendlich wollen: die des Samariters oder die des Colgate-Lächelns.

nen Zeichen und Wegmarken im diagnostischen Umgang mit den Patienten verwendet nämlich jeder von uns noch weitere berufsbezogene Symbole. Mit diesen bestimmen wir die Ausgestaltung des ärztlichen Umfelds und des Gesundheitswesens überhaupt. Sie stehen für die Art von Medizin, die wir schlussendlich wollen: die des Samariters und der professionellen Zuwendung, oder diejenige der Gesundheits-Apps, des Colgate-Lächelns oder gar des Dollarzeichens. Ja, liebe Kolleginnen und Kollegen: Nach Cassirer liegt die Verantwortung für diese gestalterische Freiheit ganz allein bei Ihnen.

\section{Bildnachweis}

Symbolbild, ( ) Hakan Kacar | Dreamstime.com. 